\title{
Singular Perturbation of Nonlinear Systems with Regular Singularity
}

\author{
Domingos H. U. Marchetti $\mathbb{D}^{1}$ and William R. P. Conti ${ }^{2}$ \\ ${ }^{1}$ Instituto de Física, Universidade de São Paulo, Rua do Matão, 1371, 05508-090 São Paulo, SP, Brazil \\ ${ }^{2}$ Departamento de Ciências do Mar, Universidade Federal de São Paulo, Rua Dr. Carvalho de Mendonça 144, \\ 11070-100 Santos, SP, Brazil
}

Correspondence should be addressed to Domingos H. U. Marchetti; marchett@if.usp.br

Received 16 April 2017; Revised 25 March 2018; Accepted 8 April 2018; Published 29 May 2018

Academic Editor: Seenith Sivasundaram

Copyright (c) 2018 Domingos H. U. Marchetti and William R. P. Conti. This is an open access article distributed under the Creative Commons Attribution License, which permits unrestricted use, distribution, and reproduction in any medium, provided the original work is properly cited.

We extend Balser-Kostov method of studying summability properties of a singularly perturbed inhomogeneous linear system with regular singularity at origin to nonlinear systems of the form $\varepsilon z f^{\prime}=F(\varepsilon, z, f)$ with $F$ a $\mathbb{C}^{\nu}$-valued function, holomorphic in a polydisc $\bar{D}_{\rho} \times \bar{D}_{\rho} \times \bar{D}_{\rho}^{v}$. We show that its unique formal solution in power series of $\varepsilon$, whose coefficients are holomorphic functions of $z$, is 1-summable under a Siegel-type condition on the eigenvalues of $F_{f}(0,0,0)$. The estimates employed resemble the ones used in KAM theorem. A simple lemma is applied to tame convolutions that appear in the power series expansion of nonlinear equations. Applications to spherical Bessel functions and probability theory are indicated. The proposed summability method has certain advantages as it may be applied as well to (singularly perturbed) nonlinear partial differential equations of evolution type.

\section{Introduction}

We consider singularly perturbed nonlinear systems of the form $\left(f^{\prime}\right.$ means derivative of $f$ with respect to $\left.z\right)$

$$
\varepsilon z f^{\prime}=F(\varepsilon, z, f) \text {, }
$$

with $f=\left(f^{1}, \ldots, f^{\nu}\right)$ and $F=\left(F^{1}, \ldots, F^{\nu}\right) \nu$-vector functions, $F^{i}$ holomorphic in a polydisc, say $\bar{D}_{\rho} \times \bar{D}_{\rho_{1}} \times \bar{D}_{\rho_{1}}^{\nu}$ for some $\rho_{1}>\rho>0$ (here, $D_{\rho}\left(z_{0}\right)=\left\{z \in \mathbb{C}:\left|z-z_{0}\right|<\rho\right\}$ denotes an open disc of radius $\rho>0$, centered at $z_{0}, \bar{D}_{\rho}\left(z_{0}\right)$ denotes its closure and $\left.D_{\rho}=D_{\rho}(0)\right)$ such that the $\nu \times v$ matrix $A_{0}=F_{f}(0,0,0)$ is invertible, a condition that makes (1) possess a regular singularity at $z=0$.

When (1) is linear, i.e., $F=A f-b$, where $b=b(\varepsilon, z)$ and $A=A(\varepsilon, z)$ are, respectively, a $\nu$-vector and a $\nu \times$ $v$ matrix, whose entries are holomorphic in the polydisc $D_{R} \times D_{R}, R>0$, such that $A(0,0)^{-1}$ exists, Balser and Kostov [1] have established the following: (a) there exists a unique formal solution in the ring $\mathcal{O}(r)[[\varepsilon]]_{1}$ of formal power series

$$
\widehat{f}(\varepsilon, z)=\sum_{i=0}^{\infty} a_{i}(z) \varepsilon^{i}
$$

in $\varepsilon$ with coefficients $a_{i}(z)$ in the ring $\mathcal{O}(r)$ of holomorphic functions on $D_{r}$, continuous in its closure, satisfying

$$
\max _{|z| \leq r}\left|a_{i}(z)\right| \leq C \mu^{i} i !, \quad i=0,1,2, \ldots
$$

for some positive constants $C, \mu$ and $0<r<R$; (b) provided the closed sector $\bar{S}(\theta, \gamma ; E)$ of opening angle $\gamma$ about the bisecting direction $\theta$ and radius $E$ does not contain any ray on the direction of the eigenvalues $\lambda_{j}$ 's of $A(0,0)$,

$$
\left|\arg \lambda_{j}-\theta\right|>\frac{\gamma}{2}, \quad j=1, \ldots, n,
$$

$\widehat{f}(\varepsilon, z)$ is the 1 -Gevrey asymptotic expansion as $\varepsilon$ tends to 0 of a holomorphic function $f(\varepsilon, z)$ in $S(\theta, \gamma ; E) \times D_{r}$; (c) if $\gamma>\pi$ is chosen so that (4) holds, then the formal series $\widehat{f}(\varepsilon, z)$ is, by an analogue of Borel-Ritt's theorem for Gevrey asymptotic expansion (see, e.g., Section 3.2 of [2]), 1-summable in the direction $\theta$ and its sum equals $f(\varepsilon, z)$. 
Summability of formal solutions to singularly perturbed linear systems with irregular singularity at $z=0$,

$$
\varepsilon z^{1+r} f^{\prime}=A f-b
$$

with the Poincaré rank $r \geq 1$, has been investigated before in [3]. Contrarily to the previous case $(r=0)$, the unique formal power series solution $\widehat{f}(\varepsilon, z)$ is always 1-summable, irrespective of whether (4), the additional condition satisfied by the eingenvalues of $A(0,0)$, holds or not. The case of $r=$ -1 , on the other hand, has been studied in [4] for $v=1$ and the summability of the formal series can be read from the properties of the initial data of (5). The case $r=0$ separates the two cases and we refer to [3] for an explanation on summability properties for each of distinct cases of simple examples in which $A$ and $b=b(z)$ are a scalar and a scalar function depending only on $z$. For recent investigations of the linear meromorphic system (5) with $r \geq 1, A(z=0) \neq 0$, and $b \equiv 0$, about summable-resurgent of the Borel transform of its highest level's reduced formal solutions and connectionto-Stokes formulas, see [5] and references therein.

In the present article, all the three statements regarding the summability of the formal series $\widehat{f}(\varepsilon, z)$, (a)-(c) above, will be extended for nonlinear differential equations (1).

The nonlinear extension of Balser-Kostov's statements has been requested by our investigation of renormalization group (RG) flow equation over probability measures in $\mathbb{R}^{N}$, starting from a measure restricted to the sphere $\mathbb{S}^{N-1}$ of radius $\sqrt{N}$, as $N$ goes to $\infty$. Let $f(\varepsilon, z)$ be the unique extension in $S(0, \gamma ; E) \times$ $D_{r}$, with $\varepsilon=2 / N$, of the meromorphic function

$$
\phi(\varepsilon, z)=\frac{i}{2 \sqrt{z}} \frac{J_{N / 2}(i \sqrt{z} N)}{J_{N / 2-1}(i \sqrt{z} N)}
$$

where $J_{\kappa}(x)$ is the Bessel function of order $\kappa$. This function is the logarithmic derivative of the Fourier-Stieltjes transform $\widehat{\sigma}^{N}(x)$ of the uniform measure $\sigma^{N}$ on the $N$-dimensional sphere of radius $\sqrt{N}$. In [6], $\phi(\varepsilon, z)$ is shown to satisfy a continued fraction of Gauss, convergent in $|z| \leq 1 / 4$ uniformly in $\varepsilon$ and this domain of analyticity is extended to the upper half-plane provided $\varepsilon$ is real. In [7], $f(\varepsilon, z)$ given by (6) is the initial data whose summability properties are conjectured to be preserved under the RG dynamics. We refer to $[6,7]$ for the statistical mechanics context.

Retrospectively, the development of summability methods in probability started with the classical Wiener's Tauberian theory (see $[8,9]$ for a concise and, respectively, extensive overviews). The most common types of summation methods called matrix methods may be used as a criterion for asymptotic distribution functions $(\bmod 1)$ of numerical sequences (see Section 7 in Chapter 1 of [10] for a review and [11] for necessary conditions on general dynamical systems, in connection with Gaussian processes). Summability methods are also used in the context of Lagrange interpolation of zeros of Jacobi polynomials and complete monotonicity of certain functions (see [12] and references therein). Related to the present work is the Borel summability of the $1 / N$ expansion for the $N$-vector statistical model at high temperatures proved in [13] (see also [14] for similar results on related models). Our investigation considers a hierarchical version of this model at the critical temperature, from a dynamical point of view.

The $N$ dependence in the argument of the r.h.s. of (6) is chosen in such way that $\phi(\varepsilon, z)$ attains, as $\varepsilon$ goes to 0 , a limit function

$$
\phi(0, z)=\frac{-1}{1+\sqrt{1+4 z}}
$$

(see Proposition 2.1 of [6]). $\phi(\varepsilon, z)$ satisfies an ordinary differential equation of Riccati type

$$
\varepsilon z \phi^{\prime}+\phi-2 z \phi^{2}+\frac{1}{2}=0
$$

which, despite being nonlinear, can be dealt with by BalserKostov's method. Equation (8) is of the form (1) with $v=$ 1 and (Statements (a)-(c) hold with $1 / 2$ in (8) replaced by $\beta(\varepsilon) / 2$ for any 1 -summable $\beta(\varepsilon)=\sum_{n \geq 0} \beta_{n} \varepsilon^{n}$ formal series in $\theta$ direction. In this case, the limit function (7) is replaced by $\left.\phi_{0}=-\beta_{0} /\left(1+\sqrt{1+4 \beta_{0} z}\right)\right)$

$$
F(\varepsilon, z, f)=-\frac{\beta(\varepsilon)}{2}-f+2 z f^{2}
$$

Balser-Kostov summability proof in [1] of the formal series solution $\widehat{f}$ does not follow the usual route by which the (formal) Borel transform $\widehat{\mathscr{B}} \hat{f}$ of $\widehat{f}$ is analytically continued along some sector of infinite radius (see, e.g., [2]). Their proof establishes instead Gevrey asymptotic expansion directly from (5), requiring for this an auxiliary lemma regarding an infinite system of linear equations of the same type whose coefficient matrix $A=A(\varepsilon, z)$ is independent of $z$. Although (1) is nonlinear, the system of infinitely many equations obtained by taking derivatives of (1) with respect to $\varepsilon$ is linear and Balser-Kostov's method carries over to equation of the form (1). To prove these statements, suitable formulas and a simple but efficient way of estimating higher power of $f$ are provided.

The layout of this paper is as follows. In Section 2 (Proposition 3), we prove existence of a unique solution of (1) in power series of $z$. In Section 3 (Proposition 6), we show that the formal power series in $\varepsilon$ solution of (1) is Gevrey of order 1. In Section 4 (Proposition 7) Gevrey asymptotic is established. Our main result, the 1-summability of the formal solution of (1), is stated in Section 5 (Theorem 9) and proved using Propositions 3, 6, and 7 of the previous sections. The main ingredient (Lemma 4) is employed to tame arbitrarily large number of convolutions arisen in the expansion of $F$ in powers of $f$. The advantage of the proposed summability method is that it can be applied to nonlinear partial differential equations of evolution type $[7,15]$.

\section{Power Series in $z$}

Under the hypothesis on $F$, the series

$$
F(\varepsilon, z, f)=\sum_{\substack{n, m=0: \\ n+m \neq 0}}^{\infty} A_{n, m}(\varepsilon) z^{n} f^{\cdot m}
$$


converges (in norm) absolutely in $\bar{D}_{\rho_{1}} \times \bar{D}_{\rho_{1}}^{\nu}$, uniformly in $\varepsilon \epsilon$ $\bar{D}_{\rho}$, with the coefficients $A_{n, m}(\varepsilon)$, regarded as a multilinear operator,

$$
\begin{aligned}
f^{m} & \in \underbrace{\mathbb{C}^{\nu} \times \cdots \times \mathbb{C}^{\nu}}_{m \text { copies }} \longmapsto A_{n, m}(\varepsilon) f^{m} \in \mathbb{C}^{\nu} \\
\left(A_{n, m}(\varepsilon) f^{m}\right)^{i} & =\sum_{i_{1}, \ldots, i_{m}=1}^{\nu} A_{n, m}^{i, i_{1}, \ldots, i_{m}}(\varepsilon) f^{i_{1}} \cdots f^{i_{m}},
\end{aligned}
$$

endowed with an operator norm induced by the Euclidean space $\mathbb{C}^{\nu}$ :

$$
\left\|A_{n, m}(\varepsilon)\right\|=\sup _{\substack{\left(v_{1}, \ldots, v_{m}\right) \in \mathbb{C}^{m v} \\ \mathrm{v}_{1} \neq 0, \ldots, v_{\mathrm{m}} \neq 0}} \frac{\left\|A_{n, m}(\varepsilon) v_{1} \cdots v_{m}\right\|}{\left\|v_{1}\right\| \cdots\left\|v_{m}\right\|},
$$

holomorphic in $D_{\rho}$ as a function of $\varepsilon$.

In (12) and from now on, $f=\left(f^{1}, \ldots, f^{\nu}\right)$ denotes a $\nu$ vector with $i$-th component $f^{i}$ and Euclidean norm $\|f\|^{2}=$ $f \cdot f=\sum_{i=1}^{v} \bar{f}^{i} f^{i}$. The product $\underbrace{f \cdots f}_{m \text {-times }}$ is denoted by $f^{m}$ to distinguish from the components $f^{i}$ s of $f$.

Since the left hand side of (1) vanishes for $z=0$, and $A_{00}(\varepsilon) \equiv 0$ is assumed in (10), a solution $f=f(\varepsilon, z)$ of $(1)$ in power series reads

$$
f(\varepsilon, z)=\sum_{k=1}^{\infty} f_{k}(\varepsilon) z^{k}
$$

Remark 1. Observe that $0=F\left(\varepsilon, 0, f_{0}\right)=\sum_{m \geq 1} A_{0, m}(\varepsilon) f_{0}^{m}$ admits a trivial solution $f_{0} \equiv 0$ for $f_{0}=f(\varepsilon, 0)$ which is unique by the implicit function theorem. For the $F$ corresponding to example (9), $A_{0,0}(\varepsilon)=-\beta(\varepsilon) / 2$ does not vanish identically but $f$ and (9) may be replaced by $\tilde{f}=$ $f+\beta / 2$ and

$$
\widetilde{F}=-(1+2 \beta z) \tilde{f}+\frac{\beta^{2}}{2} z+2 z \widetilde{f}^{2},
$$

according to which $\widetilde{A}_{0,0}(\varepsilon)=0$ and $\widetilde{f}(\varepsilon, 0)=0$ hold. Equations (1), under the hypothesis of invertible $F_{f}(0,0,0)$, can always be reduced to the same form with $F$ satisfying (10). For this, by the implicit function theorem, $F\left(\varepsilon, 0, f_{0}\right)=0$ can be solved for $f_{0}(\varepsilon)$ and $\widetilde{F}(\varepsilon, z, \widetilde{f})=F\left(\varepsilon, z, f_{0}+\widetilde{f}\right)$ satisfies $\widetilde{F}(\varepsilon, 0,0)=\widetilde{A}_{0,0}(\varepsilon) \equiv 0$.

Substituting the power series (14) into (10) together with (1), we are led to a system of equations

$$
\left(\varepsilon j I-A_{0,1}(\varepsilon)\right) f_{j}=g_{j}\left(\varepsilon ; f_{1}, \ldots, f_{j-1}\right)
$$

with $g_{j}=\left(g_{j}^{1}, \ldots, g_{j}^{\nu}\right)$ given by

$$
\begin{aligned}
& g_{1}^{i}(\varepsilon)=A_{1,0}^{i}(\varepsilon), \\
& g_{j}^{i}\left(\varepsilon ; f_{1}, \ldots, f_{j-1}\right) \\
& =A_{j, 0}^{i}(\varepsilon)
\end{aligned}
$$

$$
+\sum_{\substack{n \geq 0, m \geq 1: i_{1}, \ldots, i_{m}=1 \\ 2 \leq n+m \leq j}} \sum_{n, m}^{i} A_{n, i_{1}, \ldots, i_{m}}^{i(\varepsilon)}\left(f^{i_{1}} * \cdots * f^{i_{m}}\right)_{j-n}
$$

for $j \geq 2$; for any two sequences $\alpha=\left(\alpha_{k}\right)_{k \geq 1}$ and $\beta=\left(\beta_{k}\right)_{k \geq 1}$, their convolution product $\alpha * \beta=\left((\alpha * \beta)_{k}\right)_{k \geq 1}$ is a sequence defined by $(\alpha * \beta)_{1}=0$ and

$$
(\alpha * \beta)_{k}=\sum_{l=1}^{k-1} \alpha_{l} \beta_{k-l}, \quad k \geq 2 .
$$

The restriction $n+m \leq j$ in (18) results from the fact that our sequence $f^{i}=\left(f_{k}^{i}\right)_{k \geq 1}$ starts with $k=1$ and a convolution involving $m$ sequences cannot have nonvanishing component $j-n$ if $j<n+m$.

Consequently, for any $k \in \mathbb{N}$ arbitrary, (16) for $1 \leq j \leq k$ forms a closed system of $v \cdot k$ equations, involving $v \cdot k$ unknown functions which can be solved by iteration starting from

$$
f_{1}(\varepsilon)=\left(\varepsilon I-A_{0,1}(\varepsilon)\right)^{-1} A_{1,0}(\varepsilon) .
$$

If (16) for $1 \leq j \leq k-1$ and $k \geq 2$ have been solved, then

$$
f_{k}(\varepsilon)=\left(\varepsilon k I-A_{0,1}(\varepsilon)\right)^{-1} g_{k}\left(\varepsilon ; f_{1}, \ldots, f_{k-1}\right) .
$$

Regarding the inverse matrix $\left(\varepsilon k I-A_{0,1}(\varepsilon)\right)^{-1}$, we have the following.

Lemma 2 (see Lemma 1 of [1]). Suppose (4) holds with $\theta=0$ and $\lambda_{j}, j=1, \ldots, v$, eigenvalues of $A_{0,1}(0)=F_{f}(0,0,0)$. One can always find $E>0$ such that, if $k|\varepsilon| \geq(c /(c-$ 1)) $\sup _{|\varepsilon| \leq E}\left\|A_{0,1}(\varepsilon)\right\|$ for some $c>1$, the inverse matrix in (21), given by

$$
\left(\varepsilon k I-A_{0,1}(\varepsilon)\right)^{-1}=\sum_{n=0}^{\infty} \frac{1}{(\varepsilon k)^{n+1}}\left(A_{0,1}(\varepsilon)\right)^{n},
$$

is bounded and satisfies $\left\|\left(\varepsilon k I-A_{0,1}(\varepsilon)\right)^{-1}\right\| \leq c$, uniformly in $S(0, \gamma ; E)$. If $k|\varepsilon|<(c /(c-1)) \sup _{|\varepsilon| \leq E}\left\|A_{0,1}(\varepsilon)\right\|$, let $\lambda_{j}(\varepsilon)$, $j=1, \ldots, n$, the eigenvalues of $A_{0,1}(\varepsilon)$, be so that their distances from every ray $\eta=r e^{i \tau}$ intercepting $S(0, \gamma ; E)$ are bounded from below by a constant $a>0$ :

$$
\begin{aligned}
a & =\inf \left\{\left|\lambda_{j}(\varepsilon)-r e^{i \tau}\right|: 0 \leq r<\infty,|\tau| \leq \gamma, j\right. \\
& =1, \ldots, n, \varepsilon \in S(0, \gamma ; E)\} .
\end{aligned}
$$

Then,

$$
\left|\operatorname{det}\left(\varepsilon k I-A_{0,1}(\varepsilon)\right)\right|=\prod_{j=1}^{v}\left|\varepsilon k-\lambda_{j}(\varepsilon)\right| \geq a^{v}>0
$$

together with the formula $A^{-1}=\operatorname{Adj}(A) / \operatorname{det} A$ for inverse of a matrix $A$, where $\operatorname{Adj}(A)$ is the transposed of the cofactors matrix of $A$ (see, e.g., [16]) and the boundedness in $S(0, \gamma ; E)$ of all cofactors of $A_{0,1}(\varepsilon)$ gives

$$
\left\|\left(\varepsilon k I-A_{0,1}(\varepsilon)\right)^{-1}\right\| \leq c,
$$

uniformly in $\mathrm{S}(0, \gamma ; E)$ for every $k \in \mathbb{N}$. 
Proposition 3. Let $F$ be given by (10) with the eigenvalues of $A_{0,1}(0)$ obeying hypothesis (4). There exist $\gamma, E$, and $\sigma$ such that (1) has a solution $f(\varepsilon, z)$ holomorphic in $S(0, \gamma ; E) \times D_{\sigma}$. The solution $f(\varepsilon, z)$ converges, as $\varepsilon \rightarrow 0$ in the sector $S(0, \gamma ; E)$, to the unique solution $f^{*}(z)$ of $F(0, z, f)=0$ in $D_{\sigma}$ satisfying $f(0)=0$.

Proof. Since (14) solves (1), its coefficients $f_{k}(\varepsilon)$ satisfy the formal relations (16) whose solution depends on the existence of inverse matrix $\left(\varepsilon k I-A_{0,1}(\varepsilon)\right)^{-1}$ for every $k \in \mathbb{N}$ and $\varepsilon \in S(0, \gamma, E)$. Assuming (4) holds for every eigenvalue of $A_{0,1}(0)$, let $\gamma$ and $E$ be such that (23), and consequently (25), holds. Hence, $f_{k}(\varepsilon)$ given by $(21)$ is bounded uniformly in $S(0, \gamma ; E)$, uniquely defined for every $k \in \mathbb{N}$ and, in view of these, holomorphic in $S(0, \gamma ; E)$.

Let $\phi_{l}$ and $\alpha_{n, m}$ be the supremum in $S(0, \gamma ; E)$ of $\left\|f_{l}(\varepsilon)\right\|$ and $\left\|A_{n, m}(\varepsilon)\right\|$, respectively:

$$
\begin{aligned}
\phi_{l} & =\sup _{\varepsilon \in S(0, \gamma ; E)}\left\|f_{l}(\varepsilon)\right\| \\
\alpha_{n, m} & =\sup _{\varepsilon \in S(0, \gamma ; E)}\left\|A_{n, m}(\varepsilon)\right\| .
\end{aligned}
$$

By Cauchy formula

$$
\begin{aligned}
& \frac{1}{n ! m !} F^{(0, n, m)}(\varepsilon, 0,0)\left(\frac{f}{\|f\|}\right)^{\cdot m} \\
& \quad=\frac{1}{(2 \pi i)^{2}} \oint \oint \frac{F(\varepsilon, \zeta, \phi f /\|f\|)}{\zeta^{n+1} \phi^{m+1}} d \zeta d \phi
\end{aligned}
$$

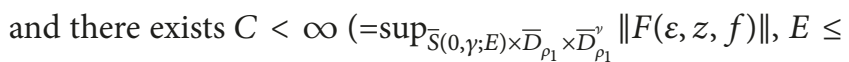
$\rho)$ such that

$$
\alpha_{n, m} \leq \frac{C}{\rho_{1}^{n} \rho_{1}^{m}} .
$$

Now, we prove that the majorant series $\sum_{l=1}^{\infty} \phi_{l} \sigma^{l}$ converges and is bounded by $\rho$ for some $0<\sigma<\rho<\rho_{1}$. For this, the following lemma will play an important role here and in the further sections. See Lemma 3.1 of [17] for similar result and Lemma 2.1 of Treves [18].

Lemma 4. Let $\lambda \geq 0$ be given and let $A=\left(1+\pi^{2} / 3\right)^{-1} / 2=$ $0.1165536 \ldots$. Consider the sequence $\left(C_{l}\right)_{l=0}^{\infty}$ with $C_{0}=A$ or $C_{0}=0$ and

$$
C_{l}=\frac{A l !^{\lambda}}{l^{2}}, \quad \forall l \geq 1 .
$$

Then

$$
\sum_{l=0}^{m} C_{l} C_{m-l} \leq C_{m}
$$

holds for every $m \geq 0$.

Proof. Since $\left(\begin{array}{c}m \\ l\end{array}\right) \geq 1$,

$$
\frac{1}{l}+\frac{1}{m-l}=\frac{m}{l(m-l)}
$$

and $0 \leq(a-b)^{2}=2\left(a^{2}+b^{2}\right)-(a+b)^{2}$ holds for any real numbers $a$ and $b$, we have

$$
\begin{aligned}
\frac{1}{C_{m}} \sum_{l=0}^{m} C_{l} C_{m-l} & \leq A\left(2+\sum_{l=1}^{m-1} \frac{m^{2}}{l^{2}(m-l)^{2}}\right) \\
& \leq 2 A\left(1+\sum_{l=1}^{m-1}\left(\frac{1}{l^{2}}+\frac{1}{(m-l)^{2}}\right)\right) \\
& \leq 2 A\left(1+\frac{\pi^{2}}{3}\right)=1 .
\end{aligned}
$$

It thus follows from (31) with $C_{0}=0$ that

$$
\sum_{\substack{l_{1}, \ldots, l_{k} \geq 1: \\ l_{1}+\cdots+l_{k}=m}} C_{l_{1}} \cdots C_{l_{k}} \leq C_{m}
$$

holds for any $1 \leq k \leq m$ and the sum is equal to 0 for $k>m$.

Let $c$ and $A$ be as in Lemmas 2 and 4. Let $C$ and $\rho_{1}$ in (29) be such that (see Remark 5)

$$
\begin{aligned}
& \alpha_{0, m} \leq \frac{\alpha A}{c \rho^{m}}, \quad m \geq 1 \\
& \alpha_{n, m} \leq \frac{\alpha}{c} C_{n} \frac{1}{\rho^{n+m}}, \quad n \geq 1, m \geq 0
\end{aligned}
$$

for some $\alpha>0$ and $0<\rho<\rho_{1}$. Suppose

$$
\phi_{l} \leq \alpha C_{l} \frac{1}{\kappa^{l}}
$$

holds for $l \geq 1$, with $\left(C_{l}\right)_{l \geq 1}$ the sequence in Lemma 4 with $\lambda=0$ and $\kappa<\rho$.

Hence, by (20) together with (25), (27), and (36), we have

$$
\phi_{1} \leq c \alpha_{1,0} \leq \alpha A \frac{1}{\kappa}
$$

and, by (21) and (18) together with (25),

$$
\begin{gathered}
\left\|f_{k}(\varepsilon)\right\| \leq c\left\|A_{k, 0}(\varepsilon)\right\|+c \sum_{\substack{n \geq 0, m \geq 1: \\
2 \leq n+m \leq k}}\left\|A_{n, m}(\varepsilon)\right\| \\
\cdot(\underbrace{\|f(\varepsilon)\| * \cdots *\|f(\varepsilon)\|}_{m})_{k-n}
\end{gathered}
$$

with $\|f(\varepsilon)\|$ denoting the sequence $\left(\left\|f_{j}(\varepsilon)\right\|\right)_{j \geq 1}$. Taking the sup over $\varepsilon \in S(0, \gamma ; E)$ in both sides together with (27), (34), and (36),

$$
\begin{aligned}
\phi_{k} & \leq c \alpha_{k, 0}+c \sum_{1 \leq m \leq k}(\alpha_{\cdot, m} * \underbrace{\phi * \cdots * \phi}_{m})_{k} \\
& \leq \alpha C_{k}\left(\frac{1}{\rho^{k}}+\sum_{m \geq 1}\left(\frac{\alpha}{\rho}\right)^{m} \frac{1}{\kappa^{k}}\right) \\
& \leq \alpha C_{k}\left(\frac{\kappa^{k}}{\rho^{k}}+\frac{\alpha}{\rho-\alpha}\right) \frac{1}{\kappa^{k}} \leq \alpha C_{k} \frac{1}{\kappa^{k}}
\end{aligned}
$$


holds for $k \geq 2$, provided $\alpha<\rho / 2$ and

$$
\kappa=\rho \sqrt{1-\frac{\alpha}{\rho-\alpha}}
$$

With $\alpha$ and $\kappa$ satisfying these conditions, we conclude

$$
\phi_{l}=\sup _{\varepsilon \in S(0, \gamma ; E)}\left\|f_{l}(\varepsilon)\right\| \leq \alpha \frac{A}{l^{2}} \frac{1}{\kappa^{l}}, \quad \forall l \geq 1
$$

and $\left(f_{l}(\varepsilon) z^{l}\right)_{l \geq 1}$ is a sequence of holomorphic functions, uniformly bounded in $S(0, \gamma ; E) \times D_{\sigma}$ by $\left(\phi_{l} \sigma^{l}\right)_{l \geq 1}$, whose sum $f(\varepsilon, z)=\sum_{l=1}^{\infty} f_{l}(\varepsilon) z^{l}$ is bounded (in norm) by

$$
\sum_{l=1}^{\infty} \phi_{l} \sigma^{l}=\frac{\alpha A \kappa}{\kappa-\sigma}=\rho
$$

provided $\sigma<\kappa$ satisfies $\sigma=\kappa(\rho-\alpha A) / \rho=$ $(\rho-\alpha A) \sqrt{1-\alpha /(\rho-\alpha)}$, by (41). Under this choice of $\sigma$, $F\left(\varepsilon, z, D_{\rho}^{\nu}\right) \subset D_{\sigma}^{\nu}$ uniformly in $S(0, \gamma ; E) \times D_{\sigma}$ and the solution we have obtained by the formal expansions (16) and (18) acquires sense. The power series solution (14) of (1) thus converges to a unique analytic function $f(\varepsilon, z)$ in $S(0, \gamma ; E) \times$ $D_{\sigma}$. The proof of uniqueness will be omitted.

From the uniform convergence of (14) we conclude that, for any fixed $z \in D_{\sigma}$, the solution $f(\varepsilon, z)$ tends to

$$
\begin{aligned}
f(0, z) & =\lim _{S(0, \gamma ; E) \ni \varepsilon \rightarrow 0} \sum_{l=0}^{\infty} f_{l}(\varepsilon) z^{l} \\
& =\sum_{l=0}^{\infty} \lim _{S(0, \gamma ; E) \ni \varepsilon \rightarrow 0} f_{l}(\varepsilon) z^{l}=f^{*}(z)
\end{aligned}
$$

where $f^{*}(z)$ is the unique solution of equation

$$
F(0, z, f)=0
$$

for $f$, by the analytic implicit function theorem (see, e.g., Section 2.3 of [19]). Note that the solution $f^{*}(z)$ is regular at $z=0$ since, by (14), it must satisfy $f(0)=0$ and this concludes the proof of Proposition 3 .

Remark 5. As observed at end of Section 1 of [1], the estimate $\sigma$ for the (uniformly in $\varepsilon \in S(0, \gamma ; E)$ ) radius of convergence of (14) can be much smaller than the radius of the largest disk in which det $A_{0,1}(0, z) \neq 0$, the radius of convergence for the solution of the linear system $F(0, z, 0)+F_{f}(0, z, 0) f=0$ at $\varepsilon=0$. The Cauchy majorant method (see Section 3.3 of [19]) applied to (45) yields a majorant

$$
\phi(z)=\frac{\rho_{1}}{2}\left(1-\sqrt{\frac{1-z / \sigma_{1}}{1-z / \rho_{1}}}\right)
$$

of $f^{*}(z)$, holomorphic in a disc $D_{\sigma_{1}}$, where $\sigma_{1}<\rho_{1}$ depends on $\left\|A_{0,1}(0)^{-1}\right\| \cdot C$, with $C$ a constant defined in (29), and $\rho_{1}$. Another (not sharp) method of this type, exploiting Lemma 4 to eliminate convolutions, yields a majorant holomorphic in a disc $D_{\sigma}$ of radius possibly smaller than $\sigma_{1}$. Despite this, since the latter method is undeniably practical and more suitable for extensions, we shall apply it in all further sections.

\section{Formal Power Series in $\varepsilon$}

As in (10), the double series

$$
F(\varepsilon, z, f)=\sum_{n, m} B_{n, m}(z) \varepsilon^{n} f^{m}
$$

converges (in norm) absolutely in $\bar{D}_{\rho} \times \bar{D}_{\rho}^{v}$, uniformly in $z \in \bar{D}_{\rho_{1}}$, with the coefficients $B_{n, m}(\varepsilon)$ regarded as a multilinear operator $f^{m} \in \mathbb{C}^{m v} \mapsto B_{n, m}(z) f^{m} \in \mathbb{C}^{\nu}$

$$
\left(B_{n, m}(z) f^{m}\right)^{i}=\sum_{i_{1}, \ldots, i_{m}=1}^{v} B_{n, m}^{i, i_{1}, \ldots, i_{m}}(z) f^{i_{1}} \cdots f^{i_{m}} .
$$

By consistency, $B_{00}(0)=0$ but $B_{00}(z)$ may not be identically zero. From here on, when no ambiguity arises, we drop the dot that precedes the power of $f$ in $f^{\cdot m}$, introduced to distinguish from its components $f^{i}$.

Proposition 6. Suppose the formal power series (2) satisfies (1), formally, with $F=F(\varepsilon, z, f)$ obeying the hypotheses stated after (1). Then, the coefficients $\left(a_{i}(z)\right)_{i \geq 0}$ of (2) are analytic functions of $z$ in the open disc $D_{\kappa}$ and there exist positive constants $C$ and $\mu$ such that

$$
\left\|a_{i}(z)\right\| \leq C i ! \mu^{i}
$$

holds for all $i \geq 0$ and $z \in \bar{D}_{\sigma}$, with $\sigma<\kappa<\rho$. In other words, the formal power series is of Gevrey order 1 ; that is, $\widehat{f}(\varepsilon, z) \in$ $\mathcal{O}(\sigma)[[\varepsilon]]_{1}$.

Proof. Substituting the power series (2) into (47), we are thus led to the following equations: for $i=0$, we have

$$
0=\sum_{m=0}^{\infty} B_{0, m}(z) a_{0}^{m}(z),
$$

which has already been solved for $a_{0}(z)$. Recall that $f^{*}(z)=$ $a_{0}(x)$ solves $F\left(0, z, f^{*}\right)=0$ and the coefficients $a_{0, j}$ of the power series $a_{0}(z)=\sum_{j=1}^{\infty} a_{0, j} z^{j}$ in $z$ satisfy

$$
\left\|a_{0, j}\right\| \leq \alpha C_{j} \frac{1}{\kappa^{j}}
$$

as the estimates for $\phi_{j}$ in the previous section, uniformly in $\varepsilon \in S(0, \gamma ; E)$, hold for $\varepsilon=0$. However,

$$
B_{0, m}(z)=\sum_{n=0}^{\infty} A_{n, m}(0) z^{n}
$$

together with (50) can be used, since the $A_{n, m}=A_{n, m}(0)$ do not depend on $\varepsilon$, to improve the disk $D_{\sigma}$ of convergence of the series of $a_{0}(z)$. See Remark 5 .

For $i \geq 1$, we have

$$
\begin{aligned}
z a_{i-1}^{\prime}(z)= & \sum_{m=1}^{\infty} m B_{0, m}(z) a_{0}^{m-1}(z) a_{i}(z) \\
& +\sum_{m=1}^{\infty}(B_{\cdot, m} * \underbrace{a(z) * \cdots * a(z)}_{m})_{i} .
\end{aligned}
$$


Observe that the sum over $m$ has no limit as the sequence $a(z)=\left(a_{k}(z)\right)_{k \geq 0}$ starts from $k=0$ and the convolution product, now defined by

$$
(\alpha * \beta)_{k}=\sum_{l=0}^{k} \alpha_{l} \beta_{k-l}, \quad k \geq 0
$$

for any two sequences $\alpha=\left(\alpha_{k}\right)_{k \geq 0}$ and $\beta=\left(\beta_{k}\right)_{k \geq 0}$, imposes no restriction on their number.

To isolate $a_{i}$, the largest index term in (53), we have to show that the matrix (recall $\left.B_{0,1}(0)=A_{0,1}(0)\right)$

$$
\begin{aligned}
& T_{0}(z)=B_{0,1}(z)+\sum_{m=2}^{\infty} m B_{0, m}(z) a_{0}^{m-1}=A_{01}(0)(I \\
& +A_{01}(0)^{-1} \\
& \left.\quad \cdot\left(B_{0,1}(z)-B_{0,1}(0)+\sum_{m=2}^{\infty} m B_{0, m}(z) a_{0}^{m-1}\right)\right)
\end{aligned}
$$

is invertible for every $z \in D_{\kappa}$ for some $\kappa \leq \rho$. For this, we take $\kappa$ so small that

$$
\begin{aligned}
& c \sup _{z \in D_{\kappa}(0)}\left(\left\|B_{0,1}(z)-B_{0,1}(0)\right\|\right. \\
& \left.\quad+\sum_{m=2}^{\infty} m\left\|B_{0, m}(z)\right\|\left\|a_{0}\right\|^{m-1}\right) \leq b<1
\end{aligned}
$$

and, consequently, $\left\|T_{0}(z)^{-1}\right\| \leq c /(1-b)$ holds uniformly in $D_{\kappa}(0)$.

It follows from (53) and (55) that

$$
\begin{aligned}
& a_{i}(z)=T_{0}(z)^{-1} \\
& \quad\left(z a_{i-1}^{\prime}(z)-\sum_{m=1}^{\infty}(B_{\cdot, m} * \underbrace{a(z) * \cdots * a(z)}_{m})_{i}\right)
\end{aligned}
$$

and this relation determines uniquely $a_{i}(z)$ in terms of earlier coefficients. Note that $a_{i}(z)$ is holomorphic in $D_{\kappa}$ and, by (51) and (43)

$$
\sup _{z \in D_{\kappa}(0)}\left|a_{0}(z)\right| \leq \delta A
$$

for any $\delta>0$, by letting $\kappa$ small enough. Now, to obtain an estimate on the growth rate of $\left|a_{i}(z)\right|$, let $\varphi_{i}$ denote the $i$-th Nagumo norm

$$
\left\|a_{i}\right\|_{i}:=\sup _{z \in D_{\kappa}(0)}\left(d_{\kappa}(z)\right)^{i}\left\|a_{i}(z)\right\|
$$

where $d_{\kappa}(z)=\kappa-|z|$.

of $a_{i}(z)$ and let $\beta_{n, m}$ the supremum in $D_{\kappa}$ of $\left\|B_{n, m}(z)\right\|$. The properties we shall use on Nagumo's norms is proved in [1] and references therein and are here summarized:

(1) $\|f+g\|_{k} \leq\|f\|_{k}+\|g\|_{k}$;

(2) $\|f g\|_{k+l} \leq\|f\|_{k}\|g\|_{l}$;
(3) $\left\|f^{\prime}\right\|_{k+1} \leq e(k+1)\|f\|_{k}$;

(4) $\|f\|_{k} \leq \kappa\|f\|_{k-1}$,

for any two functions $f$ and $g$ holomorphic in $D_{\kappa}$ and nonnegative integers $k, l$.

Let us assume that

$$
\varphi_{l} \leq \delta C_{l} \frac{1}{v^{l}}
$$

holds for $l=1,2, \ldots, i-1$ with $C_{l}=A l ! / l^{2}$, for some positive constants $\delta$ and $\nu$ to be determined. Similar to (29) and (36),

$$
\beta_{n, m}=\|B\|_{0} \leq \frac{C_{1}}{\rho_{1}^{n} \rho^{m}} \leq \frac{\delta(1-b)}{c} \frac{\delta C_{n}}{\rho^{n+m}}
$$

holds for some $C_{1}<\infty$ and $\rho_{1}$ large enough. Then, it follows by (57), (61), and (34) and the properties of Nagumo norms

$$
\begin{aligned}
\varphi_{i} & \leq \frac{c}{1-b}\left(\|z\|_{0}\left\|a_{i-1}^{\prime}\right\|_{i}\right. \\
& \left.+\sum_{n=1}^{i} \sum_{m=1}^{\infty} \beta_{n, m} \kappa^{n} \sum_{\substack{i_{1}, \ldots, i_{m} \geq 0: \\
i_{1}+\cdots+i_{m}=i-n}} \varphi_{i_{1}} \cdots \varphi_{i_{m}}\right) \leq \frac{c}{1-b} \\
& \cdot e \kappa i \varphi_{i-1}+\delta \frac{1}{v^{i}} \sum_{n=1}^{i} \sum_{m=1}^{\infty} C_{i-n}\left(\frac{\kappa}{\rho}\right)^{n} C_{n}\left(\frac{\delta}{\rho}\right)^{m} \\
& \leq\left(\frac{2 c}{1-b} e \kappa \nu+\frac{\delta}{\rho-\delta}\right) \delta C_{i} \frac{1}{v^{i}} \leq \delta C_{i} \frac{1}{v^{i}},
\end{aligned}
$$

where the last inequality holds provided $\delta<\rho / 2$ and

$$
\nu \leq \frac{1-b}{2 c e \kappa}\left(1-\frac{\delta}{\rho-\delta}\right)
$$

and this completes the induction:

$$
\sup _{z \in D_{\kappa}(0)}\left(d_{\kappa}(z)\right)^{l}\left|a_{l}(z)\right| \equiv\left\|a_{l}\right\|_{l} \leq \delta \frac{A l !}{l^{2}} \frac{1}{v^{l}} \quad \forall l \geq 1
$$

with $\delta$ and $\nu$ fixed so that (58) and (63) hold.

By definition (59) of Nagumo norm,

$$
\left\|a_{i}(z)\right\| \leq \frac{1}{(\kappa-\sigma)^{i}}\left\|a_{i}\right\|_{i} \leq C i ! \mu^{i}
$$

holds for all $i \geq 1$ uniformly in $\bar{D}_{\sigma}(0)$ for some $\sigma<\kappa$, with $C=\delta A$ and $\mu^{-1}=\nu(\kappa-\sigma)$, which concludes the proof of Proposition 6.

\section{Gevrey Asymptotic}

In order to set up an equation involving derivatives of $f$ with respect to $\varepsilon$, we write

$$
\begin{aligned}
\phi_{i}(\varepsilon, z) & =\frac{1}{i !} \frac{\partial^{i} f}{\partial \varepsilon^{i}}(\varepsilon, z) \\
\phi_{i}^{\prime}(\varepsilon, z) & =\frac{\partial \phi_{i}}{\partial z}(\varepsilon, z)
\end{aligned}
$$


and $\phi(\varepsilon, z)=\left(\phi_{i}(\varepsilon, z)\right)_{i \geq 0}$ for the sequence of those functions defined on $S(0, \gamma ; E) \times D_{\kappa}(0)$; analogously to (10) and (47), we write

$$
\begin{gathered}
F(\varepsilon, z, f)=\sum_{m=0}^{\infty} C_{m}(\varepsilon, z) f^{m} \\
F^{[i, 0,0]}(\varepsilon, z, f)=\sum_{m=0}^{\infty} C_{m}^{[i, 0]}(\varepsilon, z) f^{m}
\end{gathered}
$$

where $h^{[i]}$ stands for the $i$-th derivative of $h$ with respect to the first argument divided by $i$ !. The $i$-th "total derivative" of $F$ with respect to $\varepsilon$ ( $F$ depends on $\varepsilon$ explicitly and implicitly through $f$ ) can thus be written as

$$
\begin{aligned}
G_{i} & \left(\varepsilon, z, \phi_{0}, \ldots, \phi_{i}\right)=\frac{1}{i !} \frac{\partial^{i}}{\partial \varepsilon^{i}} F(\varepsilon, z, f) \\
& =\sum_{m=0}^{\infty}(C_{m}^{[\cdot, 0]}(\varepsilon, z) * \underbrace{\phi(\varepsilon, z) * \cdots * \phi(\varepsilon, z)}_{m})_{i} \\
& =T(\varepsilon, z) \phi_{i}+\widetilde{G}_{i}\left(\varepsilon, z, \phi_{0}, \ldots, \phi_{i-1}\right)
\end{aligned}
$$

where

$$
T(\varepsilon, z)=\sum_{n=0}^{\infty} \sum_{m=1}^{\infty} m A_{n, m}(\varepsilon) z^{n} \phi_{0}(\varepsilon, z)^{m-1}
$$

is a linear operator (matrix) and $\widetilde{G}_{i}\left(\varepsilon, z, \phi_{0}, \ldots, \phi_{i-1}\right)$ depends only on derivatives of $f$ with respect to $\varepsilon$ of order lower than $i$.

Differentiating (1) $i$ times with respect to $\varepsilon$, dividing by $i$ !, we have

$$
\varepsilon z \phi_{i}^{\prime}-T(\varepsilon, z) \phi_{i}=H_{i}(\varepsilon, z)
$$

for $i \geq 1$, where

$$
H_{i}(\varepsilon, z)=\widetilde{G}_{i}\left(\varepsilon, z, \phi_{0}, \ldots, \phi_{i-1}\right)-z \phi_{i-1}^{\prime}
$$

may be considered as inhomogeneous holomorphic function of $(\varepsilon, z)$ in $S(0, \gamma ; E) \times D_{\sigma}(0)$, and for $i=0$ simply (1):

$$
\varepsilon z \phi_{0}^{\prime}=F\left(\varepsilon, z, \phi_{0}\right)
$$

Proposition 7. Let $f(\varepsilon, z)$ be the unique holomorphic solution of $(1)$ on $S(0, \gamma ; E) \times D_{\sigma}(0)$ with $\sigma, \gamma$, and $E$ as in Proposition 3. There exist $0<\sigma_{1} \leq \sigma, 0<E_{1} \leq E$, and positive constants $C$ and $\mu$ such that

$$
\left\|\phi_{i}(\varepsilon, z)\right\| \leq C i ! \mu^{i}
$$

holds for all $i \geq 0$ and every point $(\varepsilon, z)$ in $S\left(0, \gamma ; E_{1}\right) \times \bar{D}_{\sigma_{1}}(0)$.

Proof. The case $i=0$ follows straightforwardly from Proposition 3. (70) which is a linear singular perturbation equation with regular singularity which can be dealt with in the following auxiliary result due to Balser-Kostov [1] (see Lemma 3 therein). For this, we drop temporarily all subindices $i$ in (70).
Let $T$ be expanded in power of $z$,

$$
T(\varepsilon, z)-t_{0}(\varepsilon)=\sum_{n=1}^{\infty} t_{n}(\varepsilon) z^{n}=S(\varepsilon, z)
$$

and consider a sequence $\left(\psi_{k}(\varepsilon, z)\right)_{k \geq 0}$ satisfying the system

$$
\begin{aligned}
& \varepsilon z \psi_{0}^{\prime}(\varepsilon, z)-t_{0}(\varepsilon) \psi_{0}(\varepsilon, z)=H(\varepsilon, z) \\
& \varepsilon z \psi_{k}^{\prime}(\varepsilon, z)-t_{0}(\varepsilon) \psi_{k}(\varepsilon, z)=S(\varepsilon, z) \psi_{k-1}(\varepsilon, z), \\
& k=1,2, \ldots
\end{aligned}
$$

By (74) and linearity, the sum over all equations in (75) yields an equation of the form (70) satisfying by the sum $\psi(\varepsilon, z)=\sum_{k=0}^{\infty} \psi_{k}(\varepsilon, z)$. We assume that $H(\varepsilon, z)$ admits an expansion

$$
H(\varepsilon, z)=\sum_{n=0}^{\infty} h_{n}(\varepsilon) z^{n}
$$

absolutely convergent for $|z| \leq \sigma$, uniformly in $S(0, \gamma ; E)$. For $H$ given by (68) and (71) this will actually be proven by induction when we resume the proof of Proposition 7 . We write, in addition, $f(z) \ll F(z)$ if $f(z)=\sum_{k=0}^{\infty} c_{k} z^{k}$ is majorized by $F(z)=\sum_{k=0}^{\infty} C_{k} z^{k}$, i.e., if $\left|c_{k}\right| \leq C_{k}$ holds for all $k$. If $f$ is a $\nu$-vector or a $\nu \times \nu$ matrix $f(z) \ll F(z)$ means majorized relation for each component. For any $h_{k}=$ $\left(h_{k}^{1}, \ldots, h_{k}^{\nu}\right) \in \mathbb{C}^{\nu}$, let $\left|h_{k}\right|$ denote the vector $\left(\left|h_{k}^{1}\right|, \ldots,\left|h_{k}^{v}\right|\right)$ and analogous notation for the matrix $\left|t_{k}\right|=\left[\left|t_{k}^{i, j}\right|\right]$.

Lemma 8. There exists a unique sequence of functions $\left(\psi_{k}(\varepsilon, z)\right)_{k \geq 0}$, holomorphic in $S\left(0, \gamma ; E_{1}\right) \times \bar{D}_{\sigma}(0)$, satisfying (75). Each $\psi_{k}(\varepsilon, z)$ has a zero of order $k$ at $z=0: \psi_{k}^{(0, k)}(\varepsilon, 0)=$ 0 , and satisfies

$$
\psi(\varepsilon, z)=\sum_{k=0}^{\infty} \psi_{k}(\varepsilon, z) \ll a(I-a \Gamma(z))^{-1} \Omega(z)
$$

where

$$
\begin{aligned}
& \Omega(z)=\sum_{n=0}^{\infty} \sup _{\varepsilon \in S(0, \gamma ; E)}\left|h_{n}(\varepsilon)\right| z^{n} \\
& \Gamma(z)=\sum_{n=0}^{\infty} \sup _{\varepsilon \in S(0, \gamma ; E)}\left|t_{n}(\varepsilon)\right| z^{n}
\end{aligned}
$$

holds for some $a<\infty$ and $\sigma$ small enough. $\psi(\varepsilon, z)$ is, in addition, the unique analytic solution in $S\left(0, \gamma ; E_{1}\right) \times D_{\sigma}(0)$ of

$$
\varepsilon z \psi^{\prime}(\varepsilon, z)-T(\varepsilon, z) \psi(\varepsilon, z)=H(\varepsilon, z)
$$

with $\psi(\varepsilon, 0)=0$.

Proof. Let us assume that $\psi_{k}(\varepsilon, z)$, as a function of $z$ and for every $\varepsilon$, has a zero of order $k$ at $z=0$ and is represented by

$$
\psi_{k}(\varepsilon, z)=\sum_{n=k}^{\infty} \omega_{n, k}(\varepsilon) z^{n}
$$


We will show that these assumptions lead to the actual solution of (75). Plugging (81) into (75) yields

$$
\begin{aligned}
& \left(\varepsilon n I-t_{0}(\varepsilon)\right) \omega_{n, 0}(\varepsilon)=h_{n}(\varepsilon), \quad n \geq 0 \\
& \left(\varepsilon n I-t_{0}(\varepsilon)\right) \omega_{n, k}(\varepsilon)=\sum_{m=k-1}^{n-1} t_{n-m}(\varepsilon) \omega_{m, k-1}(\varepsilon),
\end{aligned}
$$

for $1 \leq k \leq n$ and $n \geq 1$. Observe that, by (69) and (74), together with the fact that $\phi_{0}(\varepsilon, 0)=\sum_{j \geq 1} a_{j}(0) \varepsilon^{j}$ (recall $\left.a_{0}(0)=0\right)$,

$$
\begin{aligned}
& \varepsilon n I-t_{0}(\varepsilon)=\left(\varepsilon n I-A_{0,1}(\varepsilon)\right)(I \\
& \left.\quad-\left(\varepsilon n I-A_{0,1}(\varepsilon)\right)^{-1} \sum_{m \geq 1} m A_{0, m}(\varepsilon) \phi_{0}(\varepsilon, 0)^{m-1}\right)
\end{aligned}
$$

is invertible for every $\varepsilon \in D_{E_{1}}(0)$ if we take $E_{1} \leq E$ so small that

$$
c \sup _{\varepsilon \in \bar{D}_{E_{1}}(0)} \sum_{m=2}^{\infty} m\left\|A_{0, m}(\varepsilon)\right\|\left\|\phi_{0}(\varepsilon, 0)\right\|^{m-1} \leq d<1
$$

and $\left\|\left(\varepsilon n I-t_{0}(\varepsilon)\right)^{-1}\right\| \leq c /(1-d) \equiv a<\infty$ holds uniformly in $D_{E_{1}}(0)$.

From these relations, we have

$$
\begin{aligned}
& \psi_{0}(\varepsilon, z)=\sum_{n=0}^{\infty}\left(\varepsilon n I+t_{0}(\varepsilon)\right)^{-1} h_{n}(\varepsilon) z^{n} \\
& \psi_{k}(\varepsilon, z)=\sum_{n=k}^{\infty}\left(\varepsilon n I-t_{0}(\varepsilon)\right)^{-1} \sum_{m=k-1}^{n-1} t_{n-m}(\varepsilon) \omega_{m, k-1}(\varepsilon) \\
& \cdot z^{n}=\sum_{m=k-1}^{\infty}\left(\sum_{l=1}^{\infty}\left(\varepsilon(m+l) I-t_{0}(\varepsilon)\right)^{-1} t_{l}(\varepsilon) z^{l}\right) \\
& \cdot \omega_{m, k-1}(\varepsilon) z^{m} .
\end{aligned}
$$

Defining

$$
\Psi_{k}(z)=\sum_{n=k}^{\infty} \sup _{\varepsilon \in S\left(0, \gamma ; E_{1}\right)}\left|\omega_{n, k}(\varepsilon)\right| z^{n},
$$

it follows, by (25), (78), and (79), that

$$
\begin{aligned}
& \Psi_{0}(|z|) \leq a \Omega(|z|) \\
& \Psi_{k}(|z|) \leq a \Gamma(|z|) \Psi_{k-1}(|z|)
\end{aligned}
$$

for $k \geq 1$. Since $\psi_{k}(\varepsilon, z) \ll \Psi_{k}(z)$ for $k \geq 1$ and $\psi_{0}(\varepsilon, z) \ll$ $a \Omega(z)$ for $k=0$ hold for all $(\varepsilon, z) \in S\left(0, \gamma ; E_{1}\right) \times \bar{D}_{\sigma}(0)$, we conclude (77) provided the geometric series $\sum_{k \geq 1} a^{k}\|\Gamma(\sigma)\|^{k}$ converges for some $\sigma$. By (74) and (69)

$$
\|\Gamma(\sigma)\|=\sum_{n=1}^{\infty} \sup _{\varepsilon \in S\left(0, \gamma ; E_{1}\right)}\left\|t_{n-1}(\varepsilon)\right\| \sigma^{n}<\frac{1}{a}
$$

if $\sigma$ is chosen small enough and, thence, $\sum_{k=0}^{\infty} \psi_{k}(\varepsilon, z)=\psi(\varepsilon$, $z$ ) is a uniformly convergent series of analytic functions in $S\left(0, \gamma ; E_{1}\right) \times D_{\sigma}(0)$ which solves $(80)$. Since no other solution of (80), regular at $z=0$, exists, the proof of Lemma 8 is concluded.

We continue the proof of Proposition 7. It remains to show that the series (76) is uniformly convergent in $S\left(0, \gamma ; E_{1}\right) \times D_{\sigma}(0)$. This follows by induction. Clearly, $h_{0}(\varepsilon, z)$ is holomorphic in $S\left(0, \gamma ; E_{1}\right) \times D_{\sigma}(0)$. Suppose that $\phi_{j}(\varepsilon, z)$ is holomorphic in $S\left(0, \gamma ; E_{1}\right) \times D_{\sigma}(0)$ for each $1 \leq j<i$. Then, by $(71), h_{i}(\varepsilon, z)$ is holomorphic in the same domain. By Lemma $8, \phi_{i}(\varepsilon, z)$ is holomorphic in $S\left(0, \gamma ; E_{1}\right) \times D_{\sigma}(0)$ and, by (71), we conclude it also holds for $h_{i+1}(\varepsilon, z)$, justifying its representation as a convergent series (76), uniformly in $S\left(0, \gamma ; E_{1}\right) \times D_{\sigma}(0)$. By induction, $\phi_{i}(\varepsilon, z)$ is holomorphic in $S\left(0, \gamma ; E_{1}\right) \times D_{\sigma}(0)$ for each $i \geq 1$ and

$$
\begin{aligned}
\phi_{i}(\varepsilon, z) & \ll a(I-a \Gamma(z))^{-1} \Omega_{i}(z) \\
& \ll a(I-a \Gamma(\sigma))^{-1} \Omega_{i}(z),
\end{aligned}
$$

where $\Omega_{i}$ depends on the $\phi_{j}(\varepsilon, z)$ with $j<i$. For $i=0$, by $(78)$,

$$
|f(\varepsilon, z)|=\left|\phi_{0}(\varepsilon, z)\right| \leq a(I-a \Gamma(\sigma))^{-1} \Omega_{0}(|z|) \leq e_{0}
$$

holds for all $\varepsilon \in S\left(0, \gamma ; E_{1}\right)$ and $z \in D_{\sigma}(0)$. For $i \geq 1$, we consider the modification of Nagumo norms:

$$
\begin{aligned}
& \|f\|_{j} \\
& \quad=\sup _{z \in D_{\sigma}(0)}\left(d_{\sigma_{1}}(z)\right)^{j} \sum_{n=0}^{\infty} \sup _{\varepsilon \in S(0, \gamma ; E)} \frac{1}{n !}\left\|\frac{\partial^{n} f}{\partial z^{n}}(\varepsilon, 0)\right\||z|^{n},
\end{aligned}
$$

with $d_{\sigma}(z)=\sigma-|z|$. Recalling (71), (76), and (78), it follows from (89) that

$$
\left\|\phi_{i}\right\|_{i} \leq \frac{a}{1-a\left\|\Gamma\left(\sigma_{1}\right)\right\|}\left\|H_{i}\right\|_{i}
$$

where, by (68),

$$
\left\|H_{1}\right\|_{1} \leq \sum_{m}\left\|C_{m}^{[1,0]}\right\|_{1}\|\phi\|_{0}^{m} \leq C \frac{\|\phi\|_{0}}{\rho-\|\phi\|_{0}}
$$

and, together with the properties of Nagumo norms, for $i \geq 2$

$$
\begin{aligned}
\left\|H_{i}\right\|_{i} \leq & \|z\|_{0}\left\|\phi_{i-1}^{\prime}\right\|_{i} \\
& +\sum_{m} \sum_{\substack{i_{0}, \ldots, i_{m} \geq 0: \\
i_{0}+\cdots+i_{m}=1}}\left\|C_{m}^{\left[i_{0}, 0\right]}\right\|_{i_{0}}\left\|\phi_{i_{1}}\right\|_{i_{1}} \cdots\left\|\phi_{i_{m}}\right\|_{i_{m}} .
\end{aligned}
$$

From these, together with (92), a recursive relation of the same type studied in Section 3 may be derived for the $\left\|\phi_{l}\right\|_{l}$ (see (60)-(64)) and one may conclude that (the details for this estimate are left to the reader)

$$
\left\|\phi_{l}\right\|_{l-1} \leq \Delta \frac{A l !}{l^{2}} \omega^{l}
$$

holds for all $l \geq 1$ and some suitable constants $\Delta$ and $\omega$. Picking $\sigma_{1}<\sigma$ together with the property of Nagumo norms yields 


$$
\left\|\phi_{i}(\varepsilon, z)\right\| \leq \frac{\sigma}{\left(\sigma-\sigma_{1}\right)^{i}}\left\|\phi_{i}\right\|_{i-1} \leq C i ! \mu^{i}
$$

for all $i \geq 1$ uniformly in $S\left(0, \gamma ; E_{1}\right) \times \bar{D}_{\sigma_{1}}(0)$, with $C=\sigma \Delta A$ and $\mu=\omega /\left(\sigma-\sigma_{1}\right)$. We choose $C=\max \left(e_{0}, \sigma_{1} \Delta A\right)$ in order to include the $i=0$ case. This concludes the proof of Proposition 7.

\section{Summability of the Formal Series}

Theorem 9. Let (1) be considered with $F$ given by (10) where the eigenvalues of $A_{0,1}(0)$ obey hypothesis (4) for $(\varepsilon, z)$ in a domain $S(0, \gamma ; E) \times D_{\sigma}(0)$ with $\gamma>\pi$. Then, there exists a radius $\sigma>0$ such that for $z \in \bar{D}_{\sigma}(0)$ the formal solution $\widehat{f}(\varepsilon, z)$ is 1-summable in $\theta=0$ direction.

Proof. By Taylor's theorem

$$
\begin{aligned}
r_{I}(\varepsilon, z) & =\varepsilon^{-I}\left(f(\varepsilon, z)-\sum_{i=0}^{I-1} f_{i}(z) \varepsilon^{i}\right) \\
& =\frac{I}{\varepsilon^{I}} \int_{0}^{\varepsilon} f_{I}(\zeta, z)(\varepsilon-\zeta)^{I-1} d \zeta,
\end{aligned}
$$

where the integral is along a path from 0 to $\varepsilon$ inside $S(0, \gamma ; E)$. This, together with Proposition 7, implies

$$
\left\|r_{I}(\varepsilon, z)\right\| \leq C I ! \mu^{I}
$$

for every $I$ and $(\varepsilon, z) \in S^{\prime} \times \bar{D}_{\sigma}(0)$, with $S^{\prime}$ any proper subsector of $S(0, \gamma ; E)$. In addition, Proposition 6 states that $\widehat{f}(\varepsilon, z)$, a formal solution of $(1)$, is an element of $\mathcal{O}(\sigma)[[\varepsilon]]_{1}$ and therefore is an element of $\mathcal{O}(\sigma)[[\varepsilon]]_{1}$ for any $\sigma_{1}<\sigma$. Take now $\sigma_{1}$ and $E_{1}$ sufficiently small. Hence, by definition (see Section 1.5 of [2]), $\widehat{f}(\varepsilon, z)$ is an asymptotic expansion of order 1 , as $\varepsilon \rightarrow 0$ in the sector $S\left(0, \gamma ; E_{1}\right)$, of $f(\varepsilon, z)$, which by Proposition 3 is an analytic solution of (1) in the domain $S\left(0, \gamma ; E_{1}\right) \times \bar{D}_{\sigma_{1}}(0)$. Then, as $\gamma>\pi$, by hypothesis, $f(\varepsilon, z)$ is the only Gevrey order 1 asymptotic expandable function in $S\left(0, \gamma ; E_{1}\right)$ which has $\widehat{f}(\varepsilon, z)$ as its asymptotic expansion, and $\widehat{f}(\varepsilon, z)$ is 1-summable in $\theta=0$ direction (see, e.g., Section 3.2 of [2]).

\section{Summary of Results}

Let $\varepsilon z f^{\prime}=F(\varepsilon, z, f)$ be a singularly perturbed nonlinear system with $F=\left(F^{1}, \ldots, F^{\nu}\right)$ holomorphic in a polydisc $\bar{D}_{\rho} \times \bar{D}_{\rho_{1}} \times \bar{D}_{\rho_{1}}^{v}$ for some $\rho_{1}>\rho>0$ and the $\nu \times \nu$ matrix $A_{0}=F_{f}(0,0,0)$ invertible. In Sections 3,4 , and 5 , we have proven the following:

(a) There exists a unique formal solution in the ring $\mathscr{O}(r)[[\varepsilon]]_{1}$ of formal power series $\hat{f}(\varepsilon, z)=$ $\sum_{i=0}^{\infty} a_{i}(z) \varepsilon^{i}$ in $\varepsilon$ with coefficients $a_{i}(z)$ in the ring $\mathcal{O}(r)$ of holomorphic functions on $D_{r}$, continuous in its closure, satisfying $\max _{|z| \leq r}\left|a_{i}(z)\right| \leq$ $C \mu^{i} i !, i=0,1,2, \ldots$, for some positive constants $C, \mu$, and $0<r<R$. (b) Provided the closed sector $\bar{S}(\theta, \gamma ; E)$ of opening angle $\gamma$ about the bisecting direction $\theta$ and radius $E$ does not contain any ray on the direction of the eigenvalues $\lambda_{j}$ 's of $A(0,0)$ (the Siegel-type condition (4)), $\widehat{f}(\varepsilon, z)$ is the 1 -Gevrey asymptotic expansion as $\varepsilon$ tends to 0 of a holomorphic function $f(\varepsilon, z)$ in $S(\theta, \gamma ; E) \times D_{r}$.

(c) If $\gamma>\pi$ is chosen so that the eigenvalues $\lambda_{j}$ 's of $A(0,0)$ are Siegel-type, then the formal series $\widehat{f}(\varepsilon, z)$ is 1 -summable in the direction $\theta$ and its sum equals $f(\varepsilon, z)$.

To prove (a)-(c) we have extended Balser-Kostov summability method [1], establishing in Section 4 Gevrey asymptotic expansion directly from the equation. The ingredient for this is Lemma 8 (analogous to Lemma 3 of [1]), regarding an infinite system of linear equations of type (5) with $r=0$ and $A=A(\varepsilon, z)$ independent of $z$. Although (1) is nonlinear, a system of infinitely many linear equations is obtained by taking as many derivatives of (1) with respect to $\varepsilon$ as one wishes. Lemma 4 is the additional key ingredient that has provided to all the three statements suitable formulas and simple but efficient way of estimating higher power of $f$.

The nonlinear extension of Balser-Kostov summability method has been requested by our investigation of renormalization group (RG) flow equation over probability measures $\sigma^{N}$ in $\mathbb{R}^{N}$ as $N$ goes to $\infty$. In terms of its Fourier transform $\widehat{\sigma}^{N}(t, x)=\exp (-U(t, x))$ the initial value problem of the RG flow equation reads

$$
\begin{aligned}
U_{t}+ & \frac{1}{2}\left(\Delta U-|\nabla U|^{2}\right)+3 x \cdot \nabla U-4 U \\
& -\left.\frac{1}{2}\left(\Delta U-|\nabla U|^{2}\right)\right|_{x=0}=0, \quad(t, x) \in \mathbb{R}_{+} \times \mathbb{R}^{N}
\end{aligned}
$$

with $\widehat{\sigma}_{0}^{N}(x)=\Gamma(N / 2) J_{N / 2-1}(\sqrt{\beta N}|x|)(\sqrt{\beta N}|x| / 2)^{-N / 2-1}=$ $\exp (-U(0, x))$, the Fourier transform of the uniform measure $\sigma_{0}^{N}$ on the $N$-dimensional sphere of radius $\sqrt{N}$. We observe that the Gaussian measure $\widehat{\sigma}^{N, \text { Gauss }}(x)=\exp \left(-|x|^{2}\right)=$ $\exp \left(-U^{\text {Gauss }}(x)\right)$ is one stationary solution of the flow equation (99). The problem is to find $\beta \in(0, \infty)$ so that the initial uniform measure $\sigma_{0}^{N}$ is driven by RG flow to the Gaussian $\sigma^{N, \text { Gauss }}$ stationary point, interpreted in probability theory as the Gaussian scaling limit of nonlinear process (99), starting from the uniform measure on the $N$-sphere. The paper [6] deals with the $N=\infty$ case, for which the critical value is $\beta_{c}=4$. The large $N$ case is approached through resummation method [7] writing the Legendre transform of the RG flow and $\beta$ in formal power series expansion in $\varepsilon$.

We have used the radial coordinate $z=-|x|^{2}$ to describe the flow: $u^{N}(t, z)=U(t, \sqrt{N} x) / N$. As explained in the introduction, the result of the present paper applies to the initial condition $u^{N}(0, z)$. Let $f(\varepsilon, z)$ be the unique extension in $S(0, \gamma ; E) \times D_{r}$, with $\varepsilon=2 / N$ and $\beta=4$, of the meromorphic function

$$
\left(u^{N}(0, z)\right)^{\prime}=\frac{i}{2 \sqrt{z}} \frac{J_{N / 2}(2 i \sqrt{\beta z} N)}{J_{N / 2-1}(2 i \sqrt{\beta z} N)}=\phi(\varepsilon, z) .
$$


Then, $\phi$ satisfies the Riccati type differential equation (8), which is of the forma (1) with $v=1$ and $F$ given by (9), so the above summarized statements apply in this case. The usefulness of Balser-Kostov summability method combined with Lemma 4 is illustrated in $[7,15]$. The method when applied to the RG flow equation (99) yields existence of formal series in $\varepsilon$ together with Gevrey estimates to the coefficients, uniformly in $t$ (global existence) at the critical value $\beta_{c}(\varepsilon)$.

\section{Conflicts of Interest}

The authors declare that there are no conflicts of interest regarding the publication of this paper.

\section{Acknowledgments}

Domingos H. U. Marchetti thanks Gordon Slade and David Brydges for their hospitality at the University of British Columbia, at the time the present paper was written. William R. P. Conti was supported by FAPESP under Grant no. 07/59739-4.

\section{References}

[1] W. Balser and V. Kostov, "Singular perturbation of linear systems with a regular singularity," Journal of Dynamical and Control Systems, vol. 8, no. 3, pp. 313-322, 2002.

[2] W. Balser, From divergent power series to analytic functions, vol. 1582 of Lecture Notes in Mathematics, Springer-Verlag, Berlin, 1994.

[3] W. Balser and J. Mozo-Fernández, "Multisummability of formal solutions of singular perturbation problems," Journal of Differential Equations, vol. 183, no. 2, pp. 526-545, 2002.

[4] M. Hibino, "Borel summability of divergent solutions for singularly perturbed first-order ordinary differential equations," The Tohoku Mathematical Journal, vol. 58, no. 2, pp. 237-258, 2006.

[5] P. Remy, "Resurgence and highest levels connection-to-Stokes formulae for some linear meromorphic differencial system," Annales de la faculté de sciences de Toulouse XXVI, pp. 645-685, 2017.

[6] D. H. U. Marchetti, W. R. P. Conti, and L. F. Guidi, "Hierarchical spherical model from a geometric point of view," Journal of Statistical Physics, vol. 132, no. 5, pp. 811-838, 2008.

[7] D. H. U. Marchetti and W. R. P. Conti, Global formal series solution of a singularly perturbed first order partial differential equation: a problem in statistical mechanics, in preparation, http://www.fig.if.usp.br/ marchett/papers/formalseries8.pdf.

[8] N. H. Bingham, Probability measures on groups, vol. 1379 of Lecture Notes in Math, Springer, Berlin, Germany, 1989.

[9] J. Korevaar, Tauberian theory. A century of developments, Springer, Heidelberg, Germany, 2004.

[10] L. Kuipers and H. Niederreiter, Uniform distribution of sequences, Dover Publication Inc, Mineola, NY, USA, 2006.

[11] M. Weber, "Borel matrix," Commentationes Mathematicae Universitatis Carolinae, vol. 36, pp. 401-415, 1995.

[12] R. Askey, "Summability of Jacobi series," Transactions of the American Mathematical Society, vol. 179, pp. 71-84, 1973.

[13] J. Fröhlich, A. Mardin, and V. Rivasseau, "Borel summability of the $1 / N$ expansion for $N$-vector $[\mathrm{O}(N)$ nonlinear $\sigma]$ models,"
Communications in Mathematical Physics, vol. 86, pp. 87-110, 1982.

[14] V. Rivaseau, “Constructive tensor field theories," SIGMA 12, vol. 85, 31 pages, 2016.

[15] W. R. P. Conti, Grupo de Renormalização na Aproximação de Potencial Local para o modelo $O(N)$ de Heisenberg hierárquico: trajetória crítica e somabilidade da expansão $1 / \mathrm{N}$ [PhD thesis], Institute of Physics, University of São Paulo, 2011, http://www .teses.usp.br/teses/disponiveis/43/43134/tde-25022012-164421/ en.php.

[16] P. Lancaster and M. Tismenetsky, The Theory of Matrices : with Applications, Academic Press, New York, NY, USA, 2nd edition, 1985.

[17] T. Yamanaka, "A new higher order chain rule and Gevrey class," Annals of Global Analysis and Geometry, vol. 7, no. 3, pp. 179203, 1989.

[18] F. Treves, "An abstract nonlinear Cauchy-Kowalevska theorem," Transactions of the American Mathematical Society, vol. 150, pp. 77-92, 1970.

[19] M. S. Berger, "Nonlinearity and Functional Analysis, Lectures on nonlinear problems in mathematical analysis," in Pure and Applied Mathematics, Academic Press, New York, NY, USA, 1977. 


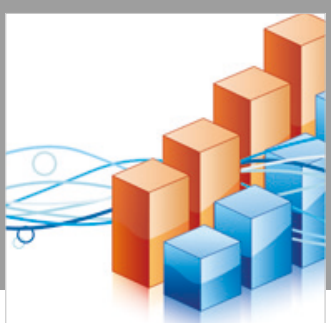

Advances in

Operations Research

\section{-n-m}
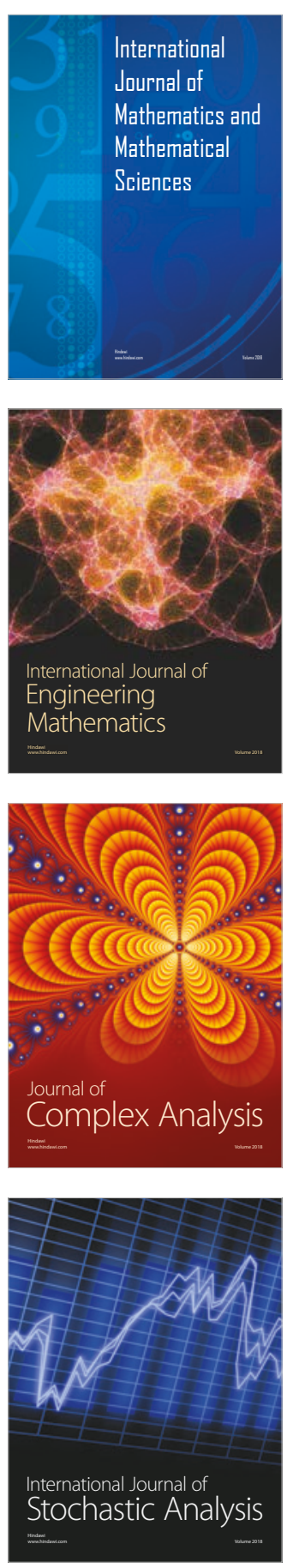
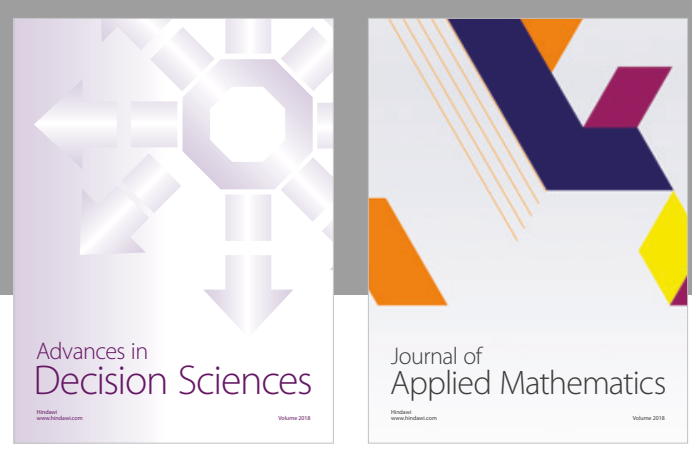

Journal of

Applied Mathematics
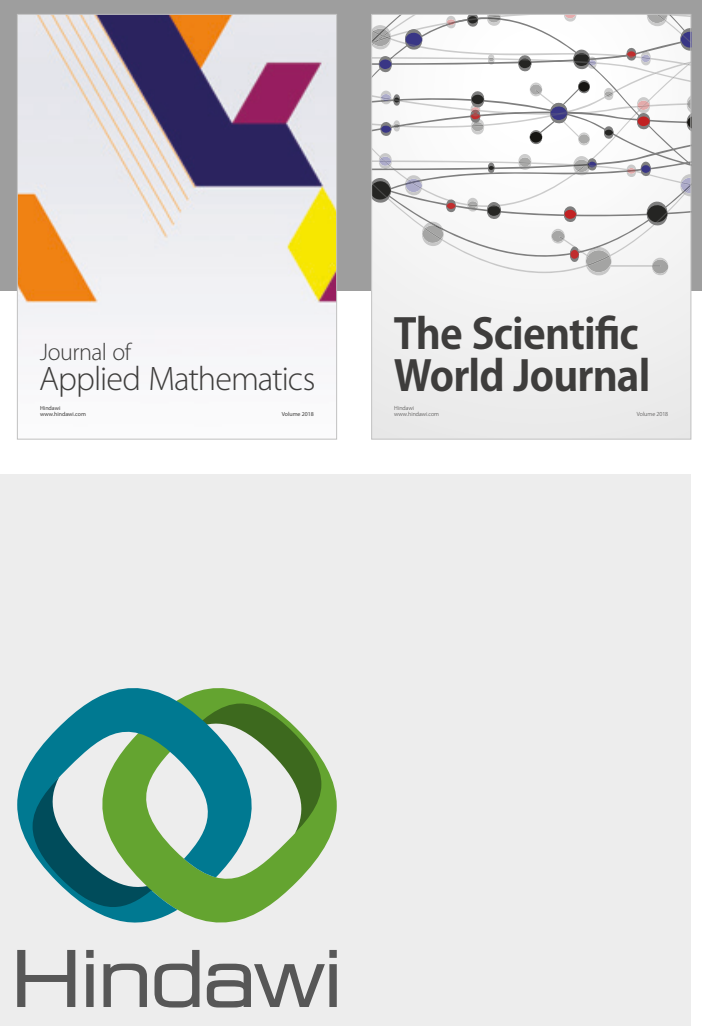

Submit your manuscripts at

www.hindawi.com

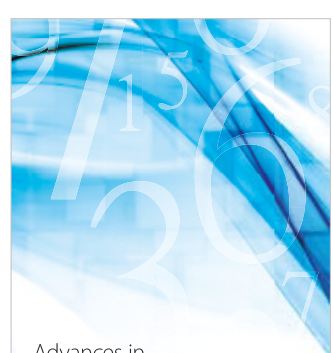

Advances in
Numerical Analysis
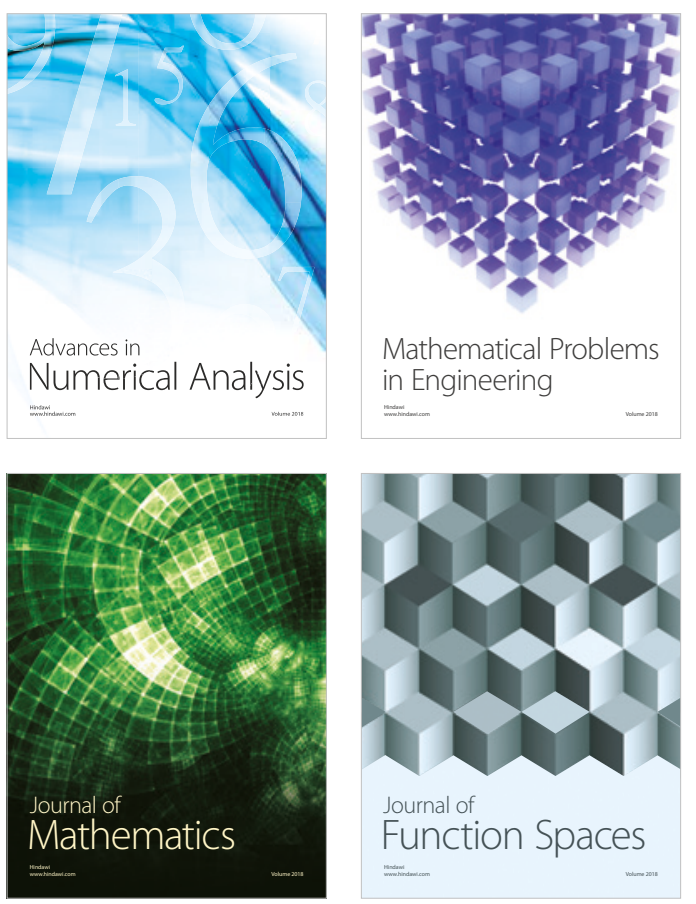

Mathematical Problems in Engineering

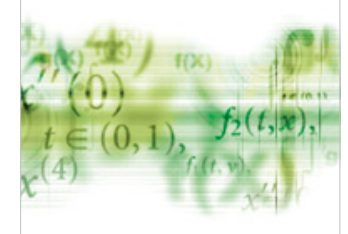

International Journal of

Differential Equations

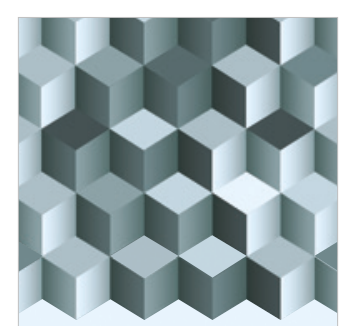

Journal of

Function Spaces

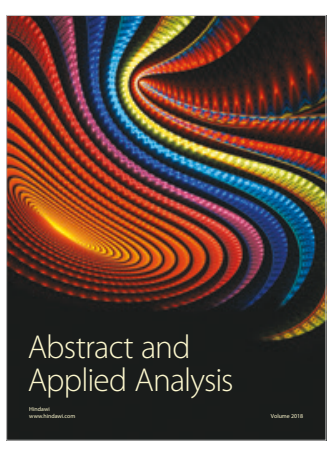

The Scientific

World Journal

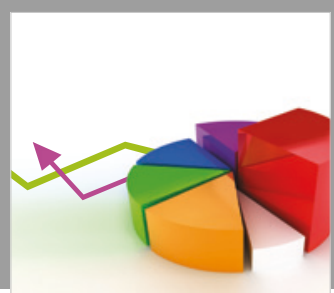

Journal of

Probability and Statistics
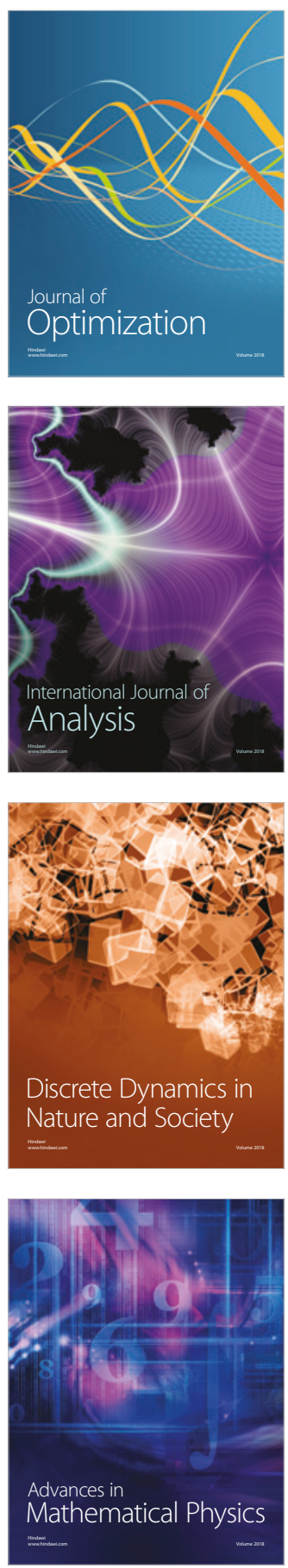